\title{
Metarhizium robertsii and $M$. acridum conidia produced on riboflavin-supplemented medium have increased UV-A tolerance and upregulated photoprotection and photoreactivation genes
}

\author{
Carla Huarte-Bonnet • Ronaldo A. Pereira-Junior • Flávia R. A. Paixão • \\ Gilberto U. L. Braga • Donald W. Roberts · Christian Luz • Nicolás Pedrini • \\ Éverton K. K. Fernandes
}

Received: 3 August 2019/Accepted: 16 December 2019/Published online: 2 January 2020

(C) International Organization for Biological Control (IOBC) 2020

\begin{abstract}
The aim of this study was to evaluate the effect of riboflavin supplementation of culture medium on conidial UV-A tolerance of $M$. acridum (Driver \& Milner) (Hypocreales: Clavicipitaceae) and $M$. robertsii (Bischoff, Rehner \& Humber) (Hypocreales: Clavicipitaceae). These fungi were produced on culture medium supplemented, or not supplemented, with riboflavin. Relative germination and expression patterns of some photoprotectionrelated genes were evaluated after irradiating with
\end{abstract}

Carla Huarte-Bonnet and Ronaldo A. Pereira-Junior have contributed equally to this work.

Handling Editor: Nicolai Meyling

Electronic supplementary material The online version of this article (https://doi.org/10.1007/s10526-019-09990-w) contains supplementary material, which is available to authorized users.

C. Huarte-Bonnet · R. A. Pereira-Junior .

F. R. A. Paixão · C. Luz · É. K. K. Fernandes $(\bowtie)$ Instituto de Patologia Tropical E Saúde Pública, Universidade Federal de Goiás, Avenida Esperança s/n, Campus Samambaia, Goiânia, GO 74690-900, Brazil e-mail: evertonkort@ufg.br

C. Huarte-Bonnet - R. A. Pereira-Junior ·

F. R. A. Paixão · N. Pedrini $(\bowtie)$

Instituto de Investigaciones Bioquímicas de La Plata -

INIBIOLP, CONICET - Facultad de Ciencias Médicas, artificial UV-A, or with filtered solar radiation (> $320 \mathrm{~nm}$; UV-A and visible radiation). M. acridum conidia harvested from riboflavin-supplemented culture medium demonstrated enhanced UV-A tolerance when irradiated with artificial UV-A. Nevertheless, relative germination of conidia of both species produced on riboflavin-supplemented medium and exposed to filtered solar radiation was significantly higher than those produced on medium not supplemented with riboflavin. Riboflavin increased the transcription of photolyases, laccases and polyketide synthase genes. However, each fungal species induced different genes patterns involved in DNA repair and photoprotection. The addition of riboflavin to the substrate used for mass production of Metarhizium spp. and the resulting enhancement of conidial tolerance to solar radiation may improve the effectiveness of these fungi in biological control programs.

Universidad Nacional de La Plata, 1900 La Plata,

Argentina

e-mail: npedrini@med.unlp.edu.ar

R. A. Pereira-Junior

Centro Universitário de Goiás - Uni-ANHANGUERA,

Goiânia, GO, Brazil

G. U. L. Braga

Departamento de Análises Clínicas, Toxicológicas E Bromatológicas, Faculdade de Ciências Farmacêuticas de Ribeirão Preto, Universidade de São Paulo,

Ribeirão Preto, SP, Brazil 
Keywords Biological control - Entomopathogenic fungi · Fungal UV tolerance · Photoprotection . Photoreactivation · Vitamin $\mathrm{B}_{2}$

\section{Introduction}

Entomopathogenic fungi such as Metarhizium spp. Sorokin, 1883 (Hypocreales: Clavicipitaceae) offer an environmentally friendly alternative to chemical pesticides. Their use as biological pesticides has been limited, however, by their relatively slow killing speed compared to chemical insecticides as well as their low tolerance to abiotic stresses, such as solar ultraviolet (UV) radiation (Braga et al. 2001a, b, c, d; Fernandes et al. 2007; Santos et al. 2011). UV radiation is part of the spectrum of electromagnetic radiation emitted by the sun and is conventionally divided in UV-A (320-400 nm), UV-B (280-320 nm) and UV-C (100-280 nm). Only UV-A and UV-B radiation reach the Earth's surface, because atmospheric ozone drastically reduces the penetration of radiation with wavelengths shorter than $320 \mathrm{~nm}$ and completely excludes those below $290 \mathrm{~nm}$ (Braga et al. 2015; Caldwell and Flint 1997). UV-A is responsible for approximately $95 \%$ of the total energy of the UV spectrum that reaches the planet surface and UV-B is responsible for the remaining 5\% (Braga et al. 2015; Schuch et al. 2017). Both UV-A and UV-B radiation have deleterious effects on Metarhizium spp. conidia, and although the effects of UV-B irradiation on conidia of entomopathogenic fungi have been studied extensively (e.g., Braga et al. 2001a, c, d, 2006; Falvo et al. 2016; Fang et al. 2010; Fernandes et al. 2007, 2015; Hunt et al. 1994; Miller et al. 2004; Nascimento et al. 2010; Pereira-Junior et al. 2018; Posadas et al. 2012; Rangel et al. 2005, 2006, 2011), information on the effects of UV-A irradiation is scarce (Braga et al. 2001c; Cadet et al. 2015; Fargues et al. 1997; Rueda Páramo et al. 2015; Yao et al. 2010).

DNA is the main target of solar UV radiation in living cells (Schuch et al. 2017). The type of DNA damage caused by the radiation depends on the wavelength of the incident UV photon (Braga et al.

D. W. Roberts

Department of Biology, Utah State University, Logan, UT, USA
2015; Douki et al. 2003; Kuluncsics et al. 1999). Damage can occur either from a direct photoreaction triggered by the absorption of UV-B or UV-A photons or by photosensitization mediated by endogenous photosensitizers (Cadet et al. 2012). UV-B is strongly absorbed by DNA while UV-A is only weakly absorbed. Thus, the direct lesion caused by the former is much more frequent than by the latter (Kuluncsics et al. 1999). Cyclobutane pyrimidine dimers (CPDs) and pyrimidine (6-4)-pyrimidone photoproducts [(6-4) photoproducts] are the two main bipyrimidine photoproducts induced directly by UV-B radiation (Fang and St. Leger 2012; Kuluncsics et al. 1999; Nascimento et al. 2010). Although UV-A constitutes a large part of UV radiation it is far less efficient in producing direct DNA photo-lesions than UV-B (Douki et al. 2003; Kuluncsics et al. 1999). Bipyrimidine photoproducts are also the main type of DNA damage induced by UV-A radiation, although produced a thousand times less efficiently than UV-B and through a mechanism different from that triggered by UV-B (Douki et al. 2003; Kuluncsics et al. 1999). UVA radiation also excites endogenous chromophores, which causes oxidative DNA damage, such as singlestrand breaks, oxidized pyrimidines and oxidized purines (essentially 8-oxo-7,8-dihydroguanine) through reactive oxygen species (Douki et al. 2003; Kuluncsics et al. 1999; Schuch et al. 2009; Yagura et al. 2017).

The two major lesions induced in DNA by UV radiation (CPDs and 6-4 photoproducts) are repaired by photolyases, which are flavoproteins that use a near-UV/blue-light photon $(300-500 \mathrm{~nm})$ as a cosubstrate (Sancar 2008). Enzymes that repair CPDs are referred to as CPD photolyase, and enzymes that repair (6-4) photoproduct are called (6-4) photolyase (Sancar 2008). All photolyases contain flavin adenine dinucleotide (FAD) group as the catalytic cofactor. Riboflavin [7,8-dimethyl-10-(1'-D-ribityl) isoalloxazine, vitamin $\mathrm{B}_{2}$ ] serves as the precursor of the flavins coenzymes such as FAD (Abbas and Sibirny 2011).

Conidia of some species can accumulate melanin or melanin-like pigments that might act as sunscreens (Braga et al. 2015). Melanins are dark pigmented multifunctional polymers formed by phenolic or indolic monomers, usually complexed with proteins and carbohydrates (Butler and Day 1998). Fungal melanins, among other functions, protect conidia 
against abiotic stresses. They are mostly synthesized via the dihydroxynaphthalene (DHN) pathway, where a polyketide synthase (PKS) catalyzes the first step in this metabolic route (Braga et al. 2015). Laccases also can be responsible for conidial pigmentation and pathogenesis. A laccase, MrLacl, was fully characterized in $M$. robertsii (=M. anisopliae s.l.) and reportedly contributes to conidial pigmentation (Fang et al. 2010). The expression of MrLacl, however, was present also in non-pigmented cells, suggesting additional roles for this enzymes.

Our recent studies were initiated in the hope to discover a simple way to improve UV-A and UV-B tolerance of fungal products destined for use in insolated field environments. We recently demonstrated that supplementing culture medium with riboflavin increased the tolerance of resulting $M$. robertsii and M. acridum (M. anisopliae var. acridum) conidia to UV-B, and this was related to increased expression of photolyase, laccase and PKS genes in these conidia (Pereira-Junior et al. 2018). The responses of Metarhizium spp. conidia produced on riboflavin-supplemented media to the tolerance to UV-A radiation or to full solar spectrum have not yet been investigated.

The current study examines the effects of riboflavin supplementation on conidia of $M$. acridum and $M$. robertsii. We evaluated if riboflavin could increase conidia tolerance to UV-A radiation emitted by UV-A fluorescent lamps and to filtered solar radiation. To study whether riboflavin could influence gene expression, we quantified transcription of genes involved in photorepair and photoprotection.

\section{Materials and methods}

Fungal isolates and culture conditions

Two isolates of Metarhizium spp. were studied: Metarhizium acridum (= M. anisopliae var. acridum) (ARSEF 324) and Metarhizium robertsii (= M. anisopliae s.l.) (ARSEF 2575), both from the USDAAgricultural Research Service Collection of Entomopathogenic Fungal Cultures of the USA (ARSEF; Robert W. Holley Center for Agriculture \& Health, USDA, Ithaca, NY, USA). Selection of these isolates was based on their known UV tolerance reported in the literature (Braga et al. 2001a, b, c, 2006; Hunt et al.
1994; Miller et al. 2004; Rangel et al. 2004, 2005). Both isolates were grown either on potato-dextroseagar medium (PDA) (Difco Laboratories, Sparks, USA) or on PDA supplemented with $0.01 \%$ (w/v) riboflavin (PDAR) (Alamar Tecno-Científica Ltda., São Paulo, Brazil), in polystyrene Petri plates $(90 \times 15 \mathrm{~mm}$, CRAL Artigos para Laboratórios Ltda., Cotia, Brazil) at $27 \pm 1{ }^{\circ} \mathrm{C}$ and $\mathrm{RH}>90 \%$, in the dark, for 15 days. PDA or PDAR plates were completely covered with a disc of $90 \mathrm{~g} \mathrm{~m}^{-2}$ sterile tracing paper (Edispel Distribuidora, Goiânia, Brazil) before fungal inoculation to prevent contamination of conidia with culture medium during their harvesting.

Conidial tolerance to UV-A radiation

Exposure to radiation from UV-A lamps in a photostability chamber

Conidial suspensions on plates, with lids removed, were exposed to $16,000 \mathrm{~mW} \mathrm{~m}^{-2}$ of UV-A radiation for 30, 60, 90 and $105 \mathrm{~min}$ at $27 \pm 1{ }^{\circ} \mathrm{C}$ in a photostability chamber (Series 6540, Caron Products \& Services Inc., Marietta, OH, USA), which is equivalent to $28.8,57.6,86.4$ and $100.8 \mathrm{~kJ} \mathrm{~m}^{-2}$ of UV-A radiation, respectively. Control plates were held in the chamber during exposure but covered with aluminum foil to block all UV radiation. The spectral irradiance (Supplemented Fig. S1a) was measured with an USB $2000+$ Rad spectroradiometer (Ocean Optics, Dunedin, FL, USA). After irradiation, plates were incubated for either $24 \mathrm{~h}$ or $48 \mathrm{~h}$ at $27 \pm 1{ }^{\circ} \mathrm{C}$ and $\mathrm{RH}>90 \%$ in the dark and then stained with Amann lactophenol with cotton blue solution.

Conidial UV-A tolerance was evaluated as previously described (Fernandes et al. 2007; Pereira-Junior et al. 2018). Conidia produced either on PDA or PDAR were harvested and suspended in $0.01 \%(\mathrm{v} / \mathrm{v})$ polyoxyethylene sorbitan monooleate (Tween 80®, Sigma Chemical Co., Saint Louis, MO, USA) solution. Suspensions were agitated, filtered through $8-\mu \mathrm{m}$ filters to remove clumps of conidia, and the concentration adjusted to $2 \times 10^{6}$ conidia $\mathrm{ml}^{-1}$. A $20 \mu \mathrm{l}$ aliquot was inoculated, without spreading, onto the center of $35 \mathrm{~mm}$ Petri plates with PDA medium supplemented with yeast extract $1 \mathrm{~g} \mathrm{l}^{-1}$ (PDAY), $0.002 \%(\mathrm{w} / \mathrm{v})$ benomyl (50\% active ingredient; Benlate ${ }^{\circ}$, DuPont, São Paulo, SP, Brazil) and 0.05\% (w/ v) chloramphenicol. Benomyl allows germination but 
kills germ tubes, and thereby prevents mycelial overgrowth that may obviate viewing conidial germination. Chloramphenicol is bactericidal. At least 300 conidia were evaluated per plate at $\times 400$ magnification, and relative percent germination was calculated according to Braga et al. (2001b). Four independent experiments were performed.

\section{Exposure to filtered solar radiation (UV-A and visible radiation)}

Additional fungal-inoculated plates were exposed to $19,126 \mathrm{~mW} \mathrm{~m}^{-2}$ filtered solar radiation for 30, 60, 90 or $120 \mathrm{~min}$ in an out-door trial, which was equivalent to $34.5,68.9,103.35$ or $137.8 \mathrm{~kJ} \mathrm{~m}^{-2}$ of UV-A radiation, respectively (supplementary Fig. S1b). During exposure, the plates were floated on water in plastic trays, with ice added as needed to avoid temperature increase, and the temperature inside one non-inoculated plate was monitored using a data logger $\left(\mathrm{HOBO}{ }^{\circledR} \mathrm{H} 8\right.$, Onset Computer Corporation, Bourne, MA, USA). The plates were covered with a $0.1 \mathrm{~mm}$-thick clear polyester film (JSC Industries, La Mirada, CA, USA) that blocks the transmission of UV wavelengths shorter than $320 \mathrm{~nm}$ (Braga et al. 2001c). Thus, conidia were exposed to solar UV-A and visible radiation. Control plates were covered with aluminum foil to block all UV radiation. After irradiation, the plates were incubated for $48 \mathrm{~h}$ at $27 \pm 1{ }^{\circ} \mathrm{C}$ and RH $>90 \%$ in the dark, and assessment of relative germination was conducted as described above. Three experiments were performed on three different sunny days in Goiânia, Goiás, Brazil [Center-West Brazil (16 40 $30^{\prime \prime} \mathrm{S}$ and $49^{\circ} 14^{\prime} 42^{\prime \prime} \mathrm{W}, 749$ m elevation)], from June 16th to July 9th, 2017, with all exposures starting at noon.

Gene transcription in Metarhizium spp. conidia exposed to UV-A and/or white light

A layer of mycelia often grew over the conidial layer on both PDA and PDAR plates. Most of this mycelial layer was removed from the culture plates with a spatula to allow irradiation of conidia, and for RNA extractions to be mostly from conidia. The culture plates were then exposed immediately to either (1) UV-A radiation in a photostability chamber at its 'UVA' setting for $105 \min \left(100.8 \mathrm{~kJ} \mathrm{~m}^{-2}\right)$, (2) white light (WL) at its 'white light' setting for $2 \mathrm{~h}$ at $16 \mathrm{Klux}$ (supplementary Fig. S1c), or (3) to UV-A followed by white light at the same doses. The WL treatment was conducted to investigate the role of possible photoreactivation. Control plates were covered with aluminum foil to block all UV-A radiation and WL. After irradiation, conidia were harvested from culture plates with a spatula and immediately macerated in liquid nitrogen with mortar and pestle. Total RNA was extracted using the RNeasy Plant Mini Kit ${ }^{\circledR}$ (Qiagen, Hilden, Germany) with an on-column DNA digestion step (DNase I, Qiagen, Hilden, Germany), following the protocol recommended by the manufacturer. Total RNA was quantified using a Nanodrop ${ }^{\circledR}$ spectrophotometer (Thermo Scientific, Wilmington, USA), and its integrity analyzed in $1 \%(\mathrm{w} / \mathrm{v})$ agarose gel. Synthesis of the first chain of cDNA was performed with the GoScript Reverse Transcription System ${ }^{\circledR}$ (Promega Corporation, Madison, USA), using 360 ng of total extracted RNA. The real-time polymerase chain reaction (q-PCR) was carried out with iQ SYBR Green Supermix (BioRad, Hercules, USA). The amplification was performed in an Mx3000P qPCR System (Stratagene, La Jolla, USA) at the following thermal conditions: denaturation at $95{ }^{\circ} \mathrm{C}$ for $10 \mathrm{~min}$, followed by 40 cycles with three-segment amplification $\left(30 \mathrm{~s}\right.$ at $95{ }^{\circ} \mathrm{C}$ for denaturation, $1 \mathrm{~min}$ at $55^{\circ} \mathrm{C}$ for annealing, and $30 \mathrm{~s}$ at $72{ }^{\circ} \mathrm{C}$ for DNA-chain elongation). The genes investigated and the primers used are listed in Table 1 . The efficiency of qPCR reaction was optimized between 85 and $115 \%$ for all primers tested. Each sample was amplified in duplicate, and three independent assays were conducted. The relative expression ratio (RER) of each target gene was calculated according to Nordgard et al. (2006) as follows:

$R E R=\frac{E_{T}^{\left.\Delta C_{t} T \text { (control-treatment }\right)}}{\sqrt{\left.E_{R 1} \Delta C_{t} R_{1} \text { (control-treatment }\right) \times E_{R 2}{ }^{\left.\Delta C_{t} R_{2} \text { (control-treatment }\right)}}}$

where $E$ is the PCR efficiency, $C_{t}$ is the cycle threshold, $R 1$ and $R 2$ are reference genes [Glyceraldehyde 3-phosphate dehydrogenase and $\gamma$-actin (Fang and Bidochka 2006)], $T$ is target gene, $\Delta C_{t}$ is the $C_{t}$ difference between the control (not irradiated) and treatments (irradiated with UV-A, WL or UVA + WL). 
Table 1 Metarhizium robertsii and Metarhizium acridum genes tested and the real-time quantitative primers sequences used

\begin{tabular}{|c|c|c|c|}
\hline Gene & Forward $\left(3^{\prime}-5^{\prime}\right)$ & Reverse $\left(5^{\prime}-3^{\prime}\right)$ & $\begin{array}{l}\text { Genebank accession } \\
\text { number/references }\end{array}$ \\
\hline \multicolumn{4}{|l|}{ Metarhizium robertsii } \\
\hline $\begin{array}{l}\text { CDP photolyase } \\
(M r P h r l)\end{array}$ & CATGCCGATTTGGACTTGCTTG & TGGAACAGCGCAATCAAACAGG & JN694762.1 \\
\hline $\begin{array}{l}\text { 6-4 photolyase } \\
(M r P h r 2)\end{array}$ & TCGCCGTTTCTTCACTTTGGTG & TCTGCGTAAACTTGGCACCGAC & JN694761.1 \\
\hline $\begin{array}{l}\text { Laccase } 1 \\
(\text { MrLacl) }\end{array}$ & CGCCTGGCTTACGTGATAC & GCTCAGGCCATGCATCAAC & XM_007825745.1 \\
\hline $\begin{array}{l}\text { Laccase } 2 \\
(M r L a c 2)\end{array}$ & TCCCTGGGTCAACGAAAGCC & CGCCGCGATAAAGTTCATGC & XM_007826363.1 \\
\hline $\begin{array}{l}\text { Laccase } 3 \\
(M r L a c 3)\end{array}$ & CTATACTTTCCGAGCCACGCAG & СССТTTCСATTCCCTCGATAGC & XM_007828431.1 \\
\hline $\begin{array}{l}\text { Polyketide synthase } 1 \\
(M r P k s 1)\end{array}$ & CATTCCGCCTCTCTCATTGCC & TGTGCGGCGCATGATATGG & XM_007825743.2 \\
\hline $\begin{array}{l}\text { Polyketide synthase } 2 \\
(M r P k s 2)\end{array}$ & CATCAGCGCCATCGGTTTAGAC & CGGGATAGGGATTGGTTTGTGG & XM_007821237.2 \\
\hline \multicolumn{4}{|l|}{ Metarhizium acridum } \\
\hline $\begin{array}{l}\text { 6-4 Photolyase } \\
(M a P h r 2)\end{array}$ & TTCATGCCGATTTGGAATTGC & TGCTTGTTTGATGGTGCCTCTG & XM_007813640.1 \\
\hline $\begin{array}{l}\text { Laccase } 1 \\
(\text { MaLacl) }\end{array}$ & AATGCGCGTACCCAACTCTG & CCACGGCGACATGTGATCTG & XM_007813533.1 \\
\hline $\begin{array}{l}\text { Laccase } 2 \\
(M a L a c 2)\end{array}$ & CGGCCAATCCACAGCTAATG & TGGCATCGGCTGTGTTTGAC & XM_007809667.1 \\
\hline $\begin{array}{l}\text { Laccase } 3 \\
(M a L a c 3)\end{array}$ & CCGCATCCCATCCACAAAC & GCTGTGGTTGGCATGGAGAC & XM_007812616.1 \\
\hline $\begin{array}{l}\text { Polyketide synthase } 1 \\
(M a P k s 1)\end{array}$ & CCGTTCCCGACTCACAATTACC & GCACAGCAAGCGGCGTTAATC & XM_007813534.1 \\
\hline $\begin{array}{l}\text { Polyketide synthase } 2 \\
(M a P k s 2)\end{array}$ & CGGCTTATCCTCCAACGAATCC & TCTGCTGCCTCTGGAAATCTCC & XM_007817459.1 \\
\hline $\begin{array}{l}\text { Reference genes (for } \\
\text { both) }\end{array}$ & & & \\
\hline Gamma actin & TCCTGACGGTCAGGTCATC & CACCAGACATGACGATGTTG & $\begin{array}{l}\text { Partial sequence } \\
\text { (Fang and Bidochka } \\
\text { 2006) }\end{array}$ \\
\hline $\begin{array}{l}\text { Glyceraldehyde } \\
\text { 3-phosphate } \\
\text { dehydrogenase }\end{array}$ & GACTGCCCGCATTGAGAAG & AGATGGAGGAGTTGGTGTTG & AY461523 \\
\hline
\end{tabular}

Statistical analysis

All data sets were previously checked for normality and homoscedasticity with Shapiro-Wilk and Barlett tests, respectively. Differences among the mean relative percent germination of conidia, and differences among the relative-gene expression ratios were determined by two-way ANOVA, which examined the effect of two independent factors: the radiation dose/time and the culture medium, followed by the Sidak's multiple comparisons test. No data transformation was required prior to ANOVA. 
$P$-values $<0.05$ were considered as significant. ANOVA followed by Sidak's multiple comparisons test were fit using GraphPad Prism ${ }^{\circledR}$ version 7.0 (GraphPad Software Inc., San Diego, CA, USA).

\section{Results}

Conidial tolerance to UV-A radiation

\section{Exposure to $U V$-A from lamps}

Conidia produced by $M$. robertsii (ARSEF 2575) and M. acridum (ARSEF 324) on PDA medium supplemented, or not, with riboflavin, exhibited a dosedependent delay in germination following UV-A exposure (Fig. 1). No significant difference in tolerance to UV-A was found for $M$. robertsii conidia produced on medium supplemented, or not, with riboflavin and exposed to radiation emitted by UV-A lamps in a photostability chamber (Fig. 1a) $(P>0.05$ for $24 \mathrm{~h}$ and $48 \mathrm{~h}$ incubation). In contrast, $M$. acridum conidia produced on riboflavin-supplemented medium were more tolerant ( $24 \mathrm{~h}$ incubation: $F_{3,24}=7.93$ and $P=0.0008 ; \quad 48 \mathrm{~h}$ incubation: $F_{3,24}=5.12$ and $P=0.007)$ to radiation showing an increased germination rate compared to conidia produced on medium without riboflavin and irradiated for $60 \mathrm{~min}$ (followed by $24 \mathrm{~h}$ and $48 \mathrm{~h}$ incubation, $P<0.01$ ) or for $105 \mathrm{~min}$ (48 h incubation, $P<0.001$ ) (Fig. 1b). Although deleterious effects were observed after 105 min irradiation, M. acridum conidia produced on riboflavinPDA supplemented medium reached similar relative germination levels as $M$. robertsii grown on either medium (Fig. 1).

\section{Exposure to solar $U V$-A and visible radiation}

In the outdoor irradiation tests, the germination of conidia produced on PDA medium supplemented with riboflavin and exposed to solar UV-A and visible radiation (wavelength $>320 \mathrm{~nm}$; see supplementary Fig. S1) was significantly higher for both $M$. robertsii and $M$. acridum isolates in comparison to conidia produced on PDA not supplemented with riboflavin [ARSEF 2575: $\left(F_{3,23}=38.00 ; P<0.0001\right)$; ARSEF 324: $\left(F_{3,23}=20.73 ; P<0.0001\right)$ ] (Fig. 2).

\section{Gene transcription}

With $M$. robertsii, transcription of CDP- and 6-4photolyase genes ( $\mathrm{MrPhrl}$ and $\mathrm{MrPhr}$ ) were higher in conidia exposed to UV-A and/or white light than the controls (MrPhrl: $\quad F_{2,12}=7.96$ and $P=0.01$; MrPhr2: $F_{2,12}=25.42$ and $\left.P<0.0001\right)$. The transcript level of MrPhrl was significantly higher $(P<0.05)$ after exposure to UV-A + WL in conidia produced on PDAR than in conidia produced on PDA only (14.2- and 2.2-fold expression, respectively) (Fig. 3a). In medium supplemented with riboflavin, $M r P h r 2$ transcript levels in conidia after exposure to UV-A and UV-A + WL (20.7- and 21.2-fold expression, respectively) were significantly higher $(P<0.001)$ than in conidia produced on PDA without riboflavin (5.1- and 1.6-fold increase, respectively). For laccases, MrLacl expression was notably higher $\left(F_{2,12}=40.41\right.$ and $\left.P<0.0001\right)$ in conidia produced on medium with riboflavin and exposed to UV-A or UV-A + WL (14.2- and 58.7-fold expression, respectively). No significant differences were observed for transcript level of MrLacl in conidia produced on PDA or PDAR and then exposed to white light alone, nor for MrLac2 and MrLac3 genes $(P>0.05$ for both MrLac2 and MrLac3) in all conditions tested (Fig. 3b). For M. robertsii polyketide synthases, the expression of MrLac2 and MrLac3 genes was increased by riboflavin supplementation after UVA + WL exposure (MrPks1: $\quad F_{2,12}=20.64$ and $P<0.0001 ; M r P k s 2: F_{2,12}=5.47$ and $\left.P=0.02\right)$.

M. acridum photolyase gene (MaPhr2) was transcriptionally induced after UV-A exposure, regardless of the media tested $\left(F_{2,12}=10.75\right.$ and $\left.P=0.002\right)$. However, the transcript level of photolyase in conidia produced on PDA was significantly higher (32.1-fold increase) than those found in conidia produced on PDAR (10.9-fold increase) (Fig. 4a). The transcript level of MaLacl was significantly higher $\left(F_{2,12}=11.47\right.$ and $\left.P=0.001\right)$ in conidia produced on PDAR than in conidia produced on PDA, after exposure to UV-A (8.7- and 1.5-fold expression, respectively) (Fig. 4b). The expression of MaLac2 and MaLac3, however, did not differ significantly ( $P>0.05$ for both genes). For all irradiation treatments, the expression of polyketide synthase genes in conidia produced on PDAR was not significantly different than those detected in conidia produced on 
Fig. 1 Relative germination of conidia of Metarhizium robertsii ARSEF 2575 (a) and $M$. acridum ARSEF 324

(b) produced on PDA medium (gray) or on PDA supplemented with $0.01 \%$ riboflavin (= PDAR)

(black), and incubated for $24 \mathrm{~h}$ and $48 \mathrm{~h}$ after being exposed to $16,000 \mathrm{~mW} \mathrm{~m}^{-2}$ of UV-A radiation for 30 , 60,90 or $105 \min (2.8,57.6$, 86.4 and $100.8 \mathrm{~kJ} \mathrm{~m}^{-2}$, respectively). Relative germination was calculated in relation to non-irradiated controls. Error bars are SE of four independent trials. Asterisks indicate significant difference between the relative germination of conidia produced on PDA or PDAR in the same incubation period: $* *(P<0.01)$, *** $(P<0.001)$
Metarhizium robertsii (ARSEF 2575)

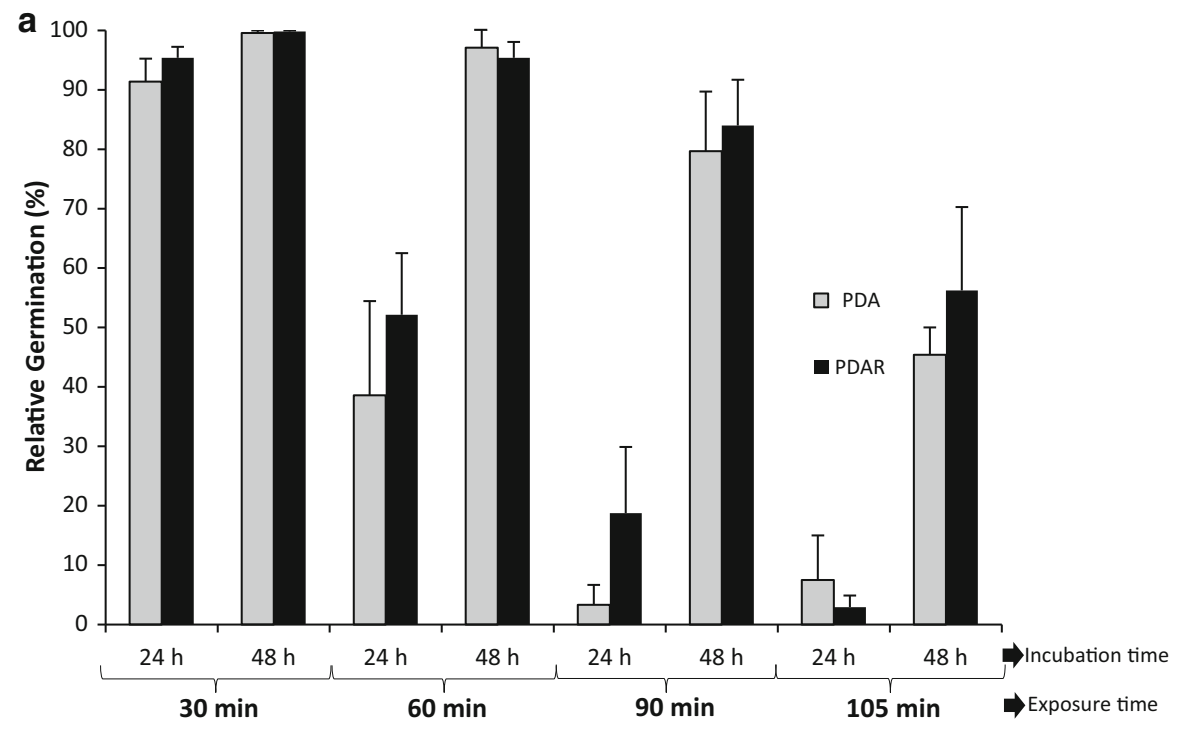

Metarhizium acridum (ARSEF 324)

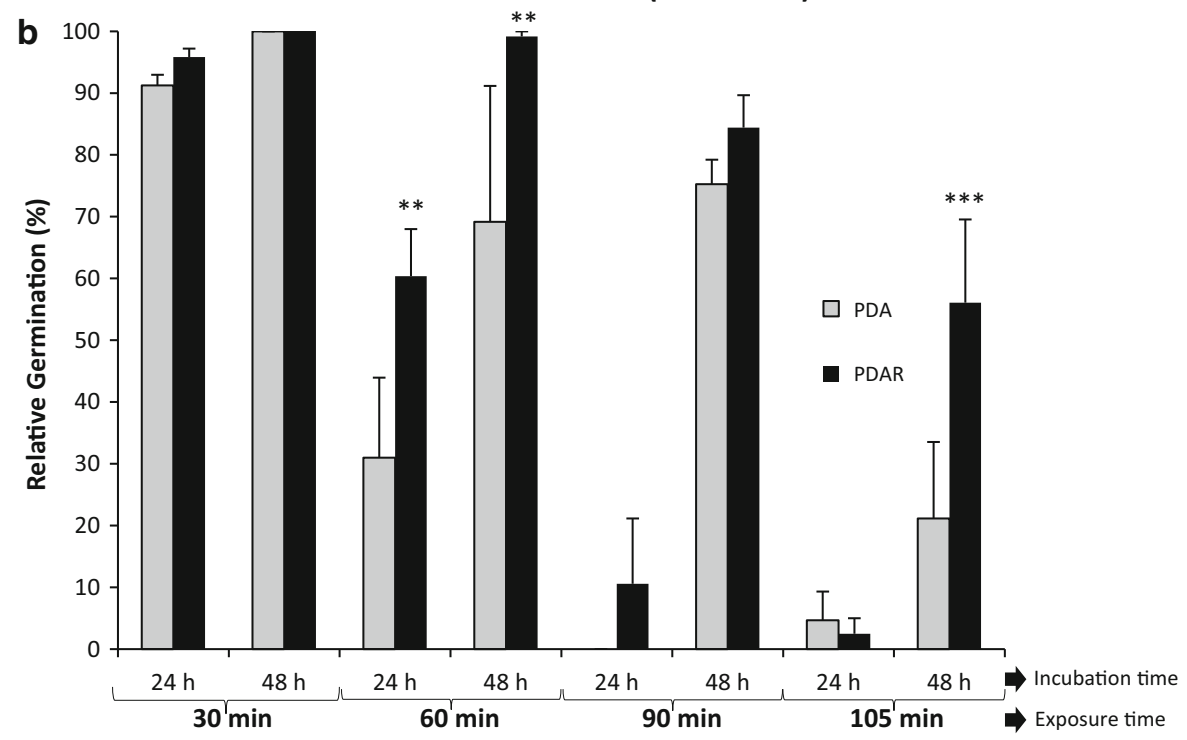

PDA ( $P>0.05$ for both MaPksl and MaPks2) (Fig. 4c).

\section{Discussion}

Riboflavin supplementation of culture medium induced M. acridum to produce conidia with increased tolerance to UV-A radiation, whether from UV-A lamps within a photostability chamber or under polyester-filtered solar radiation (wavelengths $>320 \mathrm{~nm}$ ), which include UV-A and visible radiation. However, supplementing culture media with riboflavin increased tolerance of $M$. robertsii conidia only to filtered solar radiation, but not to UV-A radiation emitted by UV-A lamps. In a recent study, we also demonstrated that growing $M$. robertsii and $M$. acridum on riboflavin-supplemented medium produced conidia with increased tolerance to UV-B radiation (Pereira-Junior et al. 2018). The effect of UVA (present study) or UV-B (Pereira-Junior et al. 2018) 

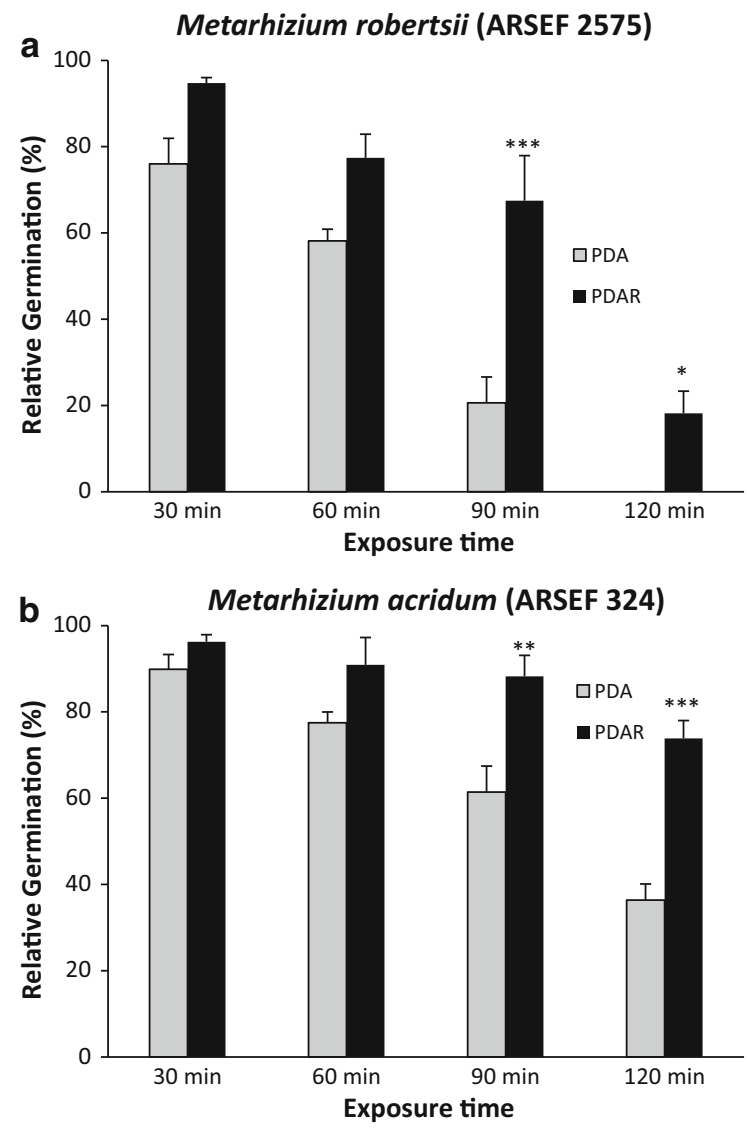

Fig. 2 Relative germination of conidia of Metarhizium robertsii ARSEF 2575 (a) and M. acridum ARSEF 324 (b) produced on PDA medium (gray), or PDA supplemented with $0.01 \%$ riboflavin (= PDAR) (black), and exposed to filtered solar UV-A radiation for $30,60,90$ or 105 min. Relative germination was calculated in relation to non-irradiated controls. Error bars are SE of four independent trials. Asterisks indicate significant difference between the relative germination of conidia produced on PDA or PDAR in the same incubation period: $*(P<0.05), * *(P<0.01), * * *(P<0.001)$

exposure on germination and gene expression of $M$. acridum and $M$. robertsii conidia produced on medium supplemented or not supplemented with riboflavin is summarized in supplementary Table S1.

Supplementation with riboflavin resulted in increased tolerance to filtered solar radiation for both M. robertsii and M. acridum conidia (Fig. 2). Results showed that the protective effect of the riboflavin supplementation against UV-A depends on the fungal strain and on the radiation used. In the case of $M$. robertsii, the difference between the tolerances of the conidia produced with or without riboflavin supplementation was much higher when filtered solar

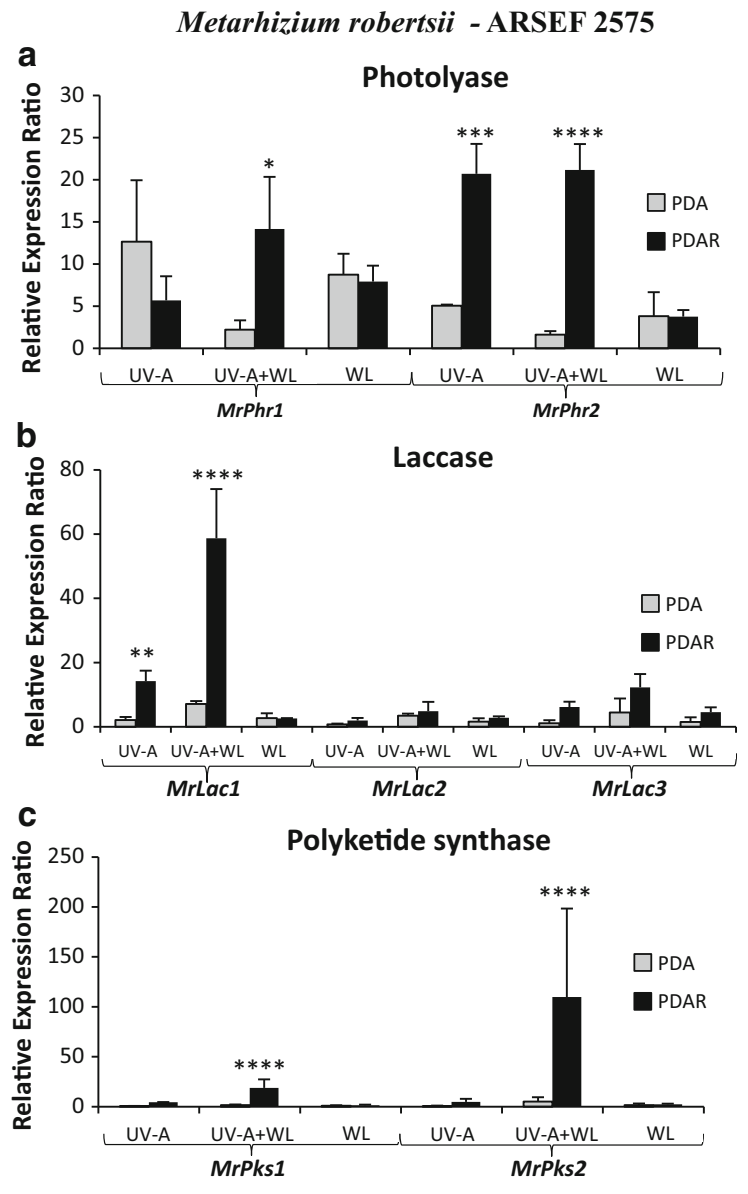

Fig. 3 Relative expression ratio of photolyase (a), laccase (b) and polyketide synthase (c) in Metarhizium robertsii (ARSEF 2575) grown on PDA medium with (PDAR) or without riboflavin (PDA) and exposed to: (1) $100.8 \mathrm{~kJ} \mathrm{~m}^{-2}$ of UV-A irradiance (UV-A), (2) UV-A $\left(100.8 \mathrm{~kJ} \mathrm{~m}^{-2}\right)$ followed by exposure to 16 Klux of white light (UV-A + WL) for $2 \mathrm{~h}$, or (3) white light only (WL) for $2 \mathrm{~h}$. Error bars are SE of three independent trials. Asterisks indicate significant difference between the gene expression of conidia produced on PDA or PDAR: $\quad *(P<0.05), \quad * *(P<0.01), \quad * * *(P<0.001)$, $* * * *(P<0.0001)$

radiation was used. In this situation, besides the UV-A radiation there is an intense visible (white) light background that improves the photoreactivation of the pyrimidines dimers by photolyases, as documented by (Chelico et al. 2005, 2006). As riboflavin is the precursor of the FAD which is the catalytic center of these enzymes, we are tempted to speculate that conidia produced on riboflavin-supplemented medium have improved photoreactivation. However, this hypothesis should be confirmed in future studies. Photoreactivation is well documented in 


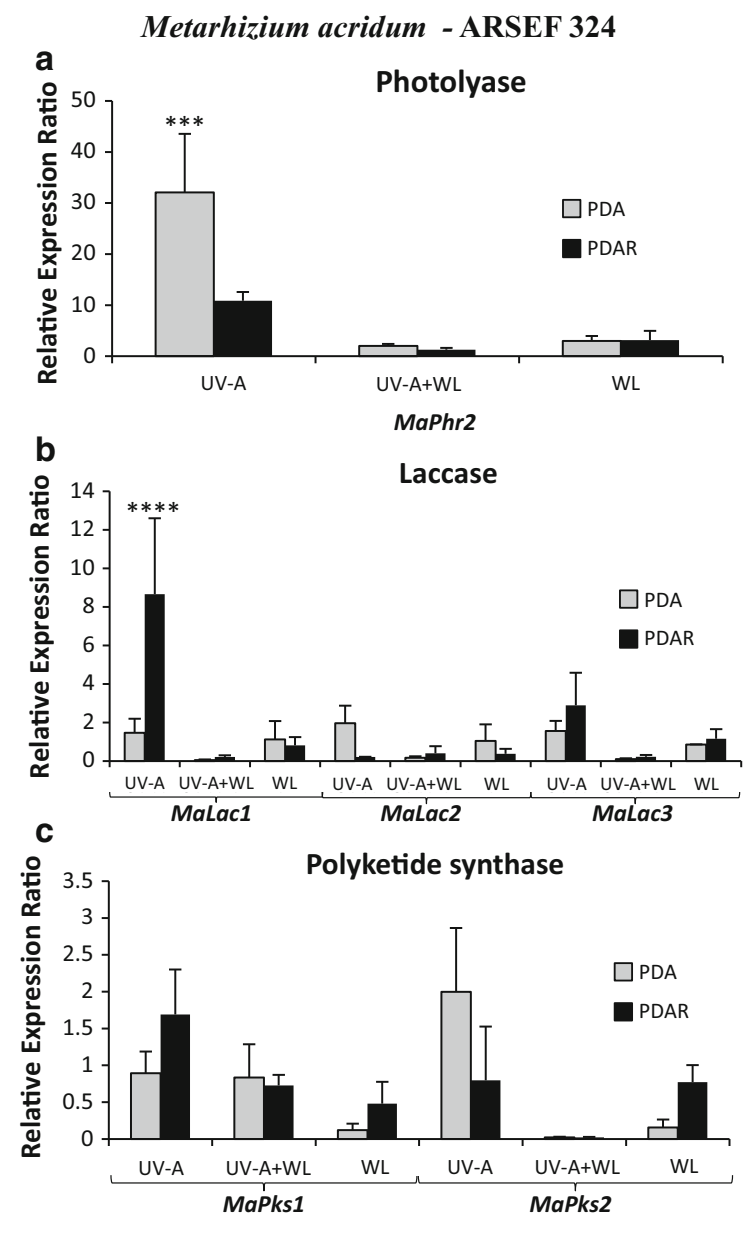

Fig. 4 Relative expression ratio of photolyase (a), laccase (b) and polyketide synthase (c) in Metarhizium acridum (ARSEF 324) grown on PDA medium with (PDAR) or without riboflavin (PDA) and exposed to: (1) $100.8 \mathrm{~kJ} \mathrm{~m}^{-2}$ of UV-A irradiance (UV-A), (2) UV-A $\left(100.8 \mathrm{~kJ} \mathrm{~m}^{-2}\right)$ followed by exposure to 16 Klux of white light (UV-A + WL) for $2 \mathrm{~h}$, or (3) white light only (WL) for $2 \mathrm{~h}$. Error bars are SE of three independent trials. Asterisks indicate significant difference between the gene expression of conidia produced on PDA or PDAR: $* * *(P<0.001), * * * *(P<0.0001)$

entomopathogenic fungi, including Metarhizium spp. (Braga et al. 2002, 2015; Chelico et al. 2005, 2006), and they are important to repair of DNA damaged by UV radiation. In the current study, transcripts of both photolyase genes ( $M r P h r 1$ and $M r P h r 2)$ increased in conidia produced on PDAR when exposed to UV-A followed by exposure to white light in the laboratory, suggesting an increase in fungal photoreactivation that correlates well with the increase in tolerance to solar radiation. Increased photoreactivation following upregulation of a photolyase gene was also demonstrated in M. acridum (Brancini et al. 2018). In fact, although UV radiation has been reported to cause DNA damage, it also contributes to the photoreactivation process (Brancini et al. 2018; Sancar 1990).

Transcription of laccases (MrLacl and MaLacl) in both fungal species as well as polyketide synthase genes (MrPks1 and MrPks2) in M. robertsii were increased in conidia exposed to either UV-A alone or followed by white light. Both laccase and polyketide synthase enzymes contribute to conidial pigmentation (Chen et al. 2015; Fang et al. 2010; Schümann and Hertweck 2006). Thus, these genes may be involved in UV protection by blocking radiation that causes cellular damage. There was significant correlation between relative expression of these genes and germination rates of $M$. robertsii conidia produced on PDAR and then exposed to UV-B radiation (Pereira-Junior et al. 2018).

Reduced viability of $M$. robertsii conidia exposed to solar UV-A at $3 \mathrm{~kJ} \mathrm{~m}^{-2}$ was reported previously, whereas there was no reduction in viability of $M$. acridum (Braga et al. 2001c). In the present study, viability (germination) of $M$. acridum conidia produced on medium enriched with riboflavin was reduced to approximately $75 \%$ after exposure to solar UV-A for $120 \mathrm{~min}$ (Fig. 2), whereas the viability was reduced to ca. 55\% when exposed to artificial UV-A for $105 \mathrm{~min}$. The literature reports that M. acridum is more tolerant to solar UV-A radiation (Braga et al. 2001c) [and also to UV-B (Braga et al. 2001a; PereiraJunior et al. 2018)] than $M$. robertsii. In these studies the fungal isolates were cultivated on PDAY, i.e., PDA supplemented with yeast extract $\left(1 \mathrm{~g} \mathrm{l}^{-1}\right)$, a component which contains B-complex vitamins, including riboflavin. In the current study, the tolerance to artificial UV-A of $M$. acridum conidia produced on PDAR medium was, at most, as high as that of $M$. robertsii. $M$. acridum, however, had higher relative mean germination than $M$. robertsii when exposed to solar UV-A radiation. These results indicate a distinctive dose-response pattern of fungal isolates to UV-A radiation, i.e., conidia that tolerate low doses might not be as tolerant to higher doses. The variation of response among fungal isolates to different UV wavelengths and irradiation doses suggest that several mechanisms might be involved in DNA damage reparation and UV photoprotection, and that different mechanisms are triggered in each isolate to mitigate UV-caused stress with different UV-A doses. In fact, it 
is well established that, in mammalian cells, UV-B induces DNA damage mainly by a direct photoreaction (thymine dimerization), whereas UV-A also damage DNA indirectly via photosensitizing reactions (Cadet et al. 2015).

The results presented in the current study, as well as our results reported previously (Pereira-Junior et al. 2018), cannot fully explain the protective effects of riboflavin to Metarhizium spp. against UV radiation. Although we measured the expression pattern of some genes potentially involved in this tolerance, other genes are likely to be involved in these pathways as well. Moreover, cross-talk between enzymes may be possible, but currently is not known. Transcriptomic and proteomics analyses might be necessary to clarify the identity and role of other genes involved in UV photoprotection and repair, and thereby provide a better understanding of UV tolerance of fungi to different UV wavelengths and doses. Hopefully, the use of riboflavin in culture media, to some degree, may produce conidia which overcome the frequently noted negative effects of environmental UV radiation on entomopathogenic fungi when used as biological control agents in field applications.

Acknowledgements We are grateful to Richard A. Humber (USDA/ARS, Ithaca, NY, USA) for providing the fungal isolates used in this study, and to Ricardo N. Marreto (Faculdade de Farmácia/UFG, Goiânia, GO, Brazil) for providing access to the Caron photostability chamber used for our artificial UV-A and white-light exposure tests. We also thank the Coordenação de Aperfeiçoamento de Pessoal de Nível Superior (CAPES) of Brazil for providing PhD scholarship for R.A. Pereira-Junior, MSc scholarship for F.R.S. Paixão, and sandwich scholarships for both R.A. Pereira-Junior and F.R.S. Paixão (PPCP CAPES/Mercosul-016/2011). This research was supported by grants from the Fundação de Amparo à Pesquisa do Estado de Goiás (FAPEG, 201210267001060), CAPES (PPCP 016/2011), Conselho Nacional de Desenvolvimento Científico e Tecnológico (CNPq) of Brazil (484329/2012-0), Instituto Nacional de Ciência e Tecnologia em Entomologia Molecular, Universidade Federal do Rio de Janeiro, Rio de Janeiro, Brazil (INCT 465678/2014-9), and Cooperative Agreements between D.W. Roberts and the United States Department of Agriculture (USDA - APHIS \#14-8130-0114 and \#14-8130-0368). We thank $\mathrm{CNPq}$ for grants $\mathrm{PQ}$ 308850/2015-7 to É.K.K. Fernandes, PQ 308189/2013-2 to C. Luz, and PQ 308505/2015-8 to G.U.L. Braga. We also thank the Consejo Nacional de Investigaciones Científicas y Técnicas (CONICET) for grant \#5055/14 to N. Pedrini. N. Pedrini is member of the CONICET Researcher's Career, Argentina.

\section{Compliance with ethical standards}

Conflict of interest The authors declare that they have no conflict of interest.

\section{References}

Abbas CA, Sibirny AA (2011) Genetic control of biosynthesis and transport of riboflavin and flavin nucleotides and construction of robust biotechnological producers. Microbiol Mol Biol Rev 75:321-360

Braga GUL, Flint SD, Messias CL, Anderson AJ, Roberts DW (2001a) Effect of UV-B on conidia and germlings of the entomopathogenic hyphomycete Metarhizium anisopliae. Mycol Res 105:874-882

Braga GUL, Flint SD, Messias CL, Anderson AJ, Roberts DW (2001b) Effects of UVB irradiance on conidia and germinants of the entomopathogenic Hyphomycete Metarhizium anisopliae: a study of reciprocity and recovery. Photochem Photobiol 73:140-146

Braga GUL, Flint SD, Miller CD, Anderson AJ, Roberts DW (2001c) Both solar UVA and UVB radiation impair conidial culturability and delay germination in the entomopathogenic fungus Metarhizium anisopliae. Photochem Photobiol 74:734-739

Braga GUL, Flint SD, Miller CD, Anderson AJ, Roberts DW (2001d) Variability in response to UV-B among species and strains of Metarhizium isolated from sites at latitudes from $61^{\circ} \mathrm{N}$ to $54^{\circ} \mathrm{S}$. J Invertebr Pathol 78:98-108

Braga GUL, Rangel DEN, Flint SD, Miller CD, Anderson AJ, Roberts DW (2002) Damage and recovery from UV-B exposure in conidia of the entomopathogens Verticillium lecanii and Aphanocladium album. Mycologia 94:912-920

Braga GUL, Rangel DEN, Flint SD, Anderson AJ, Roberts DW (2006) Conidial pigmentation is important to tolerance against solar-simulated radiation in the entomopathogenic fungus Metarhizium anisopliae. Photochem Photobiol 82:418-422

Braga GUL, Rangel DEN, Fernandes ÉKK, Flint SD, Roberts DW (2015) Molecular and physiological effects of environmental UV radiation on fungal conidia. Curr Genet 61:405-425

Brancini GTP, Bachmann L, da Ferreira MES, Rangel DEN, Braga GÚL (2018) Exposing Metarhizium acridum mycelium to visible light up-regulates a photolyase gene and increases photoreactivating ability. J Invertebr Pathol 152:35-37

Butler MJ, Day AW (1998) Fungal melanins: a review. Can J Microbiol 44:1115-1136

Cadet J, Mouret S, Ravanat JL, Douki T (2012) Photoinduced damage to cellular DNA: Direct and photosensitized reactions. Photochem Photobiol 88:1048-1065

Cadet J, Douki T, Ravanat JL (2015) Oxidatively generated damage to cellular DNA by UVB and UVA radiation. Photochem Photobiol 91:140-155 
Caldwell MM, Flint SD (1997) Uses of biological spectral weighting functions and the need of scaling for the ozone reduction problem. Plant Ecol 128:67-76

Chelico L, Haughian JL, Woytowich AE, Khachatourians GG (2005) Quantification of ultraviolet-C irradiation induced cyclobutane pyrimidine dimers and their removal in Beauveria bassiana conidiospore DNA. Mycologia 97:621-627

Chelico L, Haughian JL, Khachatourians GG (2006) Nucleotide excision repair and photoreactivation in the entomopathogenic fungi Beauveria bassiana, Beauveria brongniartii, Beauveria nivea, Metarhizium anisopliae, Paecilomyces farinosus and Verticillium lecanii. J Appl Microbiol 100:964-972

Chen Y, Feng P, Shang Y, Xu Y-J, Wang C (2015) Biosynthesis of non-melanin pigment by a divergent polyketide synthase in Metarhizium robertsii. Fungal Genet Biol 81:142-149

Douki T, Reynaud-Angelin A, Cadet J, Sage E (2003) Bipyrimidine photoproducts rather than oxidative lesions are the main type of DNA damage involved in the genotoxic effect of solar UVA radiation. Biochemistry 42:9221-9226

Falvo ML, Pereira-Junior RA, Rodrigues J, López Lastra CC, García JJ, Fernandes ÉKK, Luz C (2016) UV-B radiation reduces in vitro germination of Metarhizium anisopliae s.l. but does not affect virulence in fungus-treated Aedes aegypti adults and development on dead mosquitoes. J Appl Microbiol 121:1710-1717

Fang W, Bidochka MJ (2006) Expression of genes involved in germination, conidiogenesis and pathogenesis in $\mathrm{Me}$ tarhizium anisopliae using quantitative real-time RT-PCR. Mycol Res 110:1165-1171

Fang W, St. Leger RJ (2012) Enhanced UV resistance and improved killing of malaria mosquitoes by photolyase transgenic entomopathogenic fungi. PLoS ONE 7(8):e43069

Fang W, Fernandes ÉKK, Roberts DW, Bidochka MJ, St. Leger RJ (2010) A laccase exclusively expressed by Metarhizium anisopliae during isotropic growth is involved in pigmentation, tolerance to abiotic stresses and virulence. Fungal Genet Biol 47:602-607

Fargues J, Rougier M, Goujet R, Smits N, Coustere C, Itier B (1997) Inactivation of conidia of Paecilomyces fumosoroseus by near-ultraviolet (UVB and UVA) and visible radiation. J Invertebr Pathol 69:70-78

Fernandes ÉKK, Rangel DEN, Moraes ÁML, Bittencourt VREP, Roberts DW (2007) Variability in tolerance to UVB radiation among Beauveria spp. isolates. J Invertebr Pathol 96:237-243

Fernandes ÉKK, Rangel DEN, Braga GUL, Roberts DW (2015) Tolerance of entomopathogenic fungi to ultraviolet radiation: a review on screening of strains and their formulation. Curr Genet 61:427-440

Hunt TR, Moore D, Higgins PM, Prior C (1994) Effect of sunscreens, irradiance and resting periods on the germination of Metarhizium flavoviride conidia. Entomophaga 39:313-322

Kuluncsics Z, Perdiz D, Brulay E, Muel B, Sage E (1999) Wavelength dependence of ultraviolet-induced DNA damage distribution: involvement of direct or indirect mechanisms and possible artefacts. J Photochem Photobiol B Biol 49:71-80
Miller CD, Rangel DEN, Braga GUL, Flint S, Kwon S-I, Messias CL, Roberts DW, Anderson AJ (2004) Enzyme activities associated with oxidative stress in Metarhizium anisopliae during germination, mycelial growth, and conidiation and in response to near-UV irradiation. Can J Microbiol 50:41-49

Nascimento É, da Silva SH, dos Marques ER, Roberts DW, Braga GUL (2010) Quantification of cyclobutane pyrimidine dimers induced by UVB radiation in conidia of the fungi Aspergillus fumigatus, Aspergillus nidulans, Metarhizium acridum and Metarhizium robertsii. Photochem Photobiol 86:1259-1266

Nordgard O, Kvaløy JT, Farmen RK, Heikkila R (2006) Error propagation in relative real-time reverse transcription polymerase chain reaction quantification models: the balance between accuracy and precision. Anal Biochem 356:182-193

Pereira-Junior RA, Huarte-Bonnet C, Paixão FRS, Roberts DW, Luz C, Pedrini N, Fernandes ÉKK (2018) Riboflavin induces Metarhizium spp. to produce conidia with elevated tolerance to UV-B, and upregulates photolyases, laccases and polyketide synthases genes. J Appl Microbiol 125:159-171

Posadas JB, Angulo LM, Mini JI, Lecuona RE (2012) Natural tolerance to UV-B and assessment of photoprotectants in conidia of six native isolates of Beauveria bassiana (BalsCriv ) Vuillemin. World Appl Sci J 20:1024-1030

Rangel DEN, Braga GUL, Flint SD, Anderson AJ, Roberts DW (2004) Variations in UV-B tolerance and germination speed of Metarhizium anisopliae conidia produced on insects and artificial substrates. J Invertebr Pathol 87:77-83

Rangel DEN, Braga GUL, Anderson AJ, Roberts DW (2005) Influence of growth environment on tolerance to UV-B radiation, germination speed, and morphology of $\mathrm{Me}$ tarhizium anisopliae var. acridum conidia. J Invertebr Pathol 90:55-58

Rangel DEN, Butler MJ, Torabinejad J, Anderson AJ, Braga GUL, Day AW, Roberts DW (2006) Mutants and isolates of Metarhizium anisopliae are diverse in their relationships between conidial pigmentation and stress tolerance. J Invertebr Pathol 93:170-182

Rangel DEN, Fernandes ÉKK, Braga GUL, Roberts DW (2011) Visible light during mycelial growth and conidiation of Metarhizium robertsii produces conidia with increased stress tolerance. FEMS Microbiol Lett 315:81-86

Rueda Páramo ME, López Lastra CC, García JJ, Fernandes ÉKK, Marreto RN, Luz C (2015) Effect of ultraviolet-A radiation on the production of Leptolegnia chapmanii (Saprolegniales: Saprolegniaceae) zoospores on dead Aedes aegypti (Diptera: Culicidae) larvae and their larvicidal activity. J Invertebr Pathol 130:133-135

Sancar GB (1990) DNA photolyases: Physical properties, action mechanism, and roles in dark repair. Mutat Res - DNA Repair 236:147-160

Sancar A (2008) Structure and function of photolyase and in vivo enzymology: 50th Anniversary. J Biol Chem 283:32153-32157

Santos MP, Dias LP, Ferreira PC, Pasin LAAP, Rangel DEN (2011) Cold activity and tolerance of the entomopathogenic fungus Tolypocladium spp. to UV-B irradiation and heat. J Invertebr Pathol 108:209-213 
Schuch AP, da Galhardo RS, de Lima-Bessa KM, Schuch NJ, Menck CFM (2009) Development of a DNA-dosimeter system for monitoring the effects of pulsed ultraviolet radiation. Photochem Photobiol Sci 8:111-120

Schuch AP, Moreno NC, Schuch NJ, Menck CFM, Garcia CCM (2017) Sunlight damage to cellular DNA: Focus on oxidatively generated lesions. Free Radic Biol Med 107:110-124

Schümann J, Hertweck C (2006) Advances in cloning, functional analysis and heterologous expression of fungal polyketide synthase genes. J Biotechnol 124:690-703

Yagura T, Schuch AP, Garcia CCM, Rocha CRR, Moreno NC, Angeli JPF, Mendes D, Severino D, Bianchini Sanchez A, Di Mascio P, de Medeiros MHG, Menck CFM (2017) Direct participation of DNA in the formation of singlet oxygen and base damage under UVA irradiation. Free Radic Biol Med 108:86-93

Yao S-L, Ying S-H, Feng M-G, Hatting JL (2010) In vitro and in vivo responses of fungal biocontrol agents to gradient doses of UV-B and UV-A irradiation. BioControl $55: 413-422$

Carla Huarte-Bonnet is currently involved in developing and characterizing fungal microsclerotia-like structures.
Ronaldo A. Pereira-Junior studies formulations to improve the tolerance of fungi to UV radiation.

Flávia R. A. Paixão is dedicated to the investigation of microsclerotia formation, its tolerance to stresses, and its virulence against Aedes aegypti.

Gilberto U. L. Braga is professor of genetics and his main research interest lies on fungal photobiology and antimicrobial photodynamic treatment.

Donald W. Roberts is insect pathologist who dedicated many decades to the biological control of arthropod pests.

Christian Luz research lies on invertebrate pathology, biodiversity and formulation of entomopathogenic fungi.

Nicolás Pedrini is devoted to the study of entomopathogenic fungi and insect-host interactions.

Éverton K. K. Fernandes is professor of parasitology, with main research interest on biological control of ticks and responses of fungi to stresses. 